\title{
Crisis secesionista en Cataluña: EL MAYOR DESAFÍO AL ORDEN CONSTITUCIONAL EN ESPAÑA ${ }^{1}$
}

\section{Josep Ma. Castellà Andreu}

Universidad de Barcelona

castella@ub.edu

\section{Introducción}

El conocido como "proceso" secesionista catalán es un tema poliédrico que puede ser estudiado desde distintos puntos de vista. A lo largo de los ocho años que lleva el proceso -desde 2012 hasta ahora-, ha merecido la atención, además de la opinión pública nacional e internacional, de historiadores, filósofos de la política, sociólogos, economistas, politólogos y juristas, y, dentro de estos, de filósofos del derecho, internacionalistas, estudiosos del derecho de la Unión Europea, penalistas, procesalistas y constitucionalistas. A estas alturas, la literatura sobre el proceso independentista es ingente desde distintas perspectivas (memorias o testimonio de protagonistas más o menos relevantes, panorámicas periodísticas, estudios de historiadores y politólogos y análisis y comentarios jurídicos). A continuación, solo se pretende dar una visión general de los hechos y la interpretación jurídico-constitucional de estos que nos parece más adecuada para entender correctamente la crisis independentista. Por ello, la perspectiva de análisis que se sigue es básicamente la del derecho constitucional y, en concreto, del derecho constitucional español sobre la base de la Constitución (CE) de 1978, teniendo muy en cuenta datos y claves de explicación de orden político, sin los cuales no se tienen los elementos interpretativos suficientes de lo sucedido en Cataluña. Dicho análisis se encuadra en un marco interpretativo de fondo que es el propio de la democracia constitucional, atacada de

1 Grupo de estudios sobre Democracia y Constitucionalismo (GEDECO. Grupo consolidado, 2017, SGR 1595). El presente trabajo se inserta en el marco del proyecto financiado por el Ministerio de Ciencia e Innovación PID2019-104414GB-C32, "Instrumentos contramayoritarios en el Estado constitucional". 
frente por dicho proceso, por una parte, al emplear una visión muy reductiva de democracia, que deja de lado su ejercicio con respeto a minorías y sin las garantías de los procesos parlamentario y electoral-referendario, y, por la otra, al pretender desligar y anteponer la decisión democrática del respeto al Estado constitucional de derecho, como se irá viendo. Ello es propio de la forma de actuar de los populismos.

La sustancia política del proceso independentista -innegable- no permite considerarlo como algo ajeno al mundo jurídico. Ello es así, primero, por la llamada, de forma crítica, "judicialización” del "conflicto", que ha llevado a múltiples pronunciamientos del Tribunal Constitucional como uno de los principales instrumentos utilizados por el Gobierno del Estado para combatirlo. Incluso, se sitúa en el origen del proceso la resolución del Alto Tribunal sobre el Estatuto catalán: la sentencia 31/2010. Y, segundo, porque el acercamiento jurídico-constitucional a la cuestión de la secesión es en sí mismo necesario en un Estado constitucional de derecho.

$\mathrm{Al}$ analizar política y jurídicamente el proceso catalán, vemos cómo la invocación de la consulta popular o del referéndum como formas predominantes de ejercicio de la democracia y de la secesión o del derecho a decidir han ido y van de la mano, de modo que para decidir sobre la secesión, el instrumento adecuado se identifica con el pronunciamiento directo de la población concernida, identificada como un "demos" o sujeto político con capacidad de decidir por sí solo. Para los impulsores de la independencia, la relación entre referéndum y secesión es la de medio-fin. Para llegar al objetivo deseado de la independencia, el referéndum o la consulta popular (por ahora, sin precisar la distinción) han sido vistos como el instrumento necesario y adecuado. Para otros, sin embargo, lo fundamental era y es el "derecho a decidir" en sí mismo a través de la consulta. En este segundo caso, la secesión pasa a un segundo plano y los esfuerzos se concentran en la celebración de una consulta sobre el futuro político de Cataluña, en el cual la salida de la independencia era una de las posibles, normalmente la preferida. Unos y otros coinciden en poner el acento en la necesidad de la consulta, la cual sería el objetivo primero a alcanzar y en el que concentrarse en primer término. Este planteamiento permite encuadrar la cuestión de la secesión en la actuación del principio democrático a través de la participación directa del cuerpo electoral, lo que le da, de entrada, una fuerza legitimadora superior ante la opinión pública nacional e internacional.

En las páginas siguientes se considerará el proceso secesionista catalán de forma diacrónica, sometiendo los hechos a una interpretación crítica -que en 
parte revisa la interpretación más habitual de estos-, que surge de las instancias independentistas y que ha sido asumida por cierta literatura jurídica y politológica. Así, nos preguntaremos, primeramente, por el origen del proceso independentista y las causas que propiciaron su planteamiento: ifue la sentencia del Estatuto catalán o más bien hay que encuadrarlo en las corrientes populistas en boga en Occidente?

A continuación, se distinguirán dos fases en las que se ha desarrollado dicho proceso -la primera, soberanista (2012-2015); la segunda, independentista (2015-2017)-, atendiendo precisamente a la invocación y empleo por los promotores de la secesión, en cada fase, de categorías constitucionales distintas ya citadas: consulta o referéndum y derecho a decidir o independencia, respectivamente. En tercer lugar, reflexionaremos sobre el momento en el que nos encontramos ahora, en el cual se produce un divorcio entre la retórica independentista y los hechos, por lo general más respetuosos con el ordenamiento. Concluiremos resumiendo las salidas constitucionales que se han planteado y el juicio que nos merecen.

\section{Inicio del proceso secesionista: 2012}

Se suele identificar, sobre todo en la literatura nacionalista, el inicio del proceso con la sentencia del Tribunal Constitucional 31/2010, de 28 de junio, sobre la constitucionalidad del Estatuto catalán, aprobado en 2006, que fue impugnado, entre otros, por los diputados del Partido Popular, entonces en la oposición. La declaración de inconstitucionalidad de parte de 14 de los 223 artículos (además de disposiciones finales) que lo componen y la interpretación conforme de otros 25 preceptos habría supuesto el desmantelamiento de las opciones fundamentales del Estatuto. Esto es, habría frustrado las aspiraciones de mayor autonomía y de una posición particular de Cataluña respecto al Estatuto anterior (1979) y al resto de Comunidades Autónomas (CCAA). El Tribunal Constitucional, tras un proceso largo (4 años), polémico y en el que el órgano se dividió casi por la mitad (5 votos particulares, de los cuales 4 optaban por más declaraciones de inconstitucionalidad), revocó contenidos fundamentales de una norma adoptada por dos instituciones representativas: el Parlamento catalán, primero, y las Cortes Generales, después, y que finalmente fue ratificado por el cuerpo electoral catalán en referéndum. Ante este agravio, el pueblo catalán habría reaccionado de forma clamorosa, sobre todo en la gran manifestación del 11 de septiembre de 2012, fecha que marca simbólicamente el inicio 
del proceso (fiesta nacional de Cataluña). Esta es la narrativa predominante, que atribuye la crisis secesionista a causas endógenas relacionadas con el (mal) estado del autogobierno, sobre todo debido a la sentencia de $2010,{ }^{2}$ la cual habría puesto en crisis el mismo pacto constitucional (Pérez Royo, 2011).

Sin embargo, una mirada atenta a los hechos y a los tiempos permite poner en cuestión dicha tesis. De entrada, ciertos hechos matizan lo resumido brevemente en el párrafo anterior. Así, en primer lugar, el Estatuto de 2006 es el primer estatuto aprobado o reformado en las cortes sin consenso de las dos grandes fuerzas políticas españolas, el Partido Socialista Obrero Español (PSOE) y el Partido Popular (PP), lo que había sido la regla unánime hasta entonces dado el carácter sustancialmente constitucional de este ("Norma institucional básica de las Comunidades Autónomas", según definición del artículo 147.1 de la $\mathrm{CE}$, aunque la Constitución requiera solo la mayoría absoluta del Congreso para su aprobación, artículo 81, CE). Segundo, ello está en la base del recurso de inconstitucionalidad interpuesto por el primer partido de la oposición, que no había participado del consenso para la aprobación de este. Era la primera vez que se impugnaban las líneas maestras de un Estatuto de autonomía ante el Tribunal Constitucional. Por último, la participación en el referéndum del 17 de junio de 2006 no alcanzó siquiera el 50\% del cuerpo electoral (un 48,85\%, con un $73,9 \%$ de votos afirmativos), en contraste con el 59,7\% de los catalanes que votaron en el referéndum sobre el Estatuto anterior de 1979 (y el 88\% de votos afirmativos).

Más importante que estos últimos datos, que dan cuenta del carácter divisivo del Estatuto y que dejan entrever su origen tacticista en la confrontación partidaria, es responder a la pregunta de por qué una sentencia "contra" el

2 Como muestra autorizada de ello, véase el Preámbulo de la Resolución 5/X, aprobada por el Parlamento catalán el 23 de enero de 2013 (sobre la que luego se volverá), en el que se indica: "En los últimos años, en la vía de la profundización democrática, una mayoría de las fuerzas políticas y sociales catalanas han impulsado medidas de transformación del marco político y jurídico. La más reciente se concretó en el proceso de reforma del Estatuto de autonomía de Cataluña iniciado por el Parlamento en el año 2005. Las dificultades y negativas por parte de las instituciones del Estado español, entre las que cabe destacar la Sentencia del Tribunal Constitucional 31/2010, suponen un rechazo radical de la evolución democrática de las voluntades colectivas del pueblo catalán dentro del Estado español y crean las bases para una involución en el autogobierno, que hoy se expresa con total claridad en los aspectos políticos, competenciales, financieros, sociales, culturales y lingüísticos". Lo sustancial de esta tesis ha sido asumida incluso por autores alejados del independentismo, como los profesores de Derecho Público que elaboraron el documento en 2017 Ideas para la Reforma de la Constitución: "[...] la sentencia del Tribunal Constitucional sobre el Estatuto, [...], al margen de sus consecuencias jurídico-constitucionales, supuso un punto de inflexión en el crecimiento del independentismo en Cataluña" (Muñoz Machado et al., p. 3). 
Estatuto de junio de 2010 provoca el surgimiento de un proceso independentista en septiembre de 2012. ¿Qué ocurre en Cataluña y en toda España en este periodo de más de dos años? Responder correctamente esta pregunta proporciona claves certeras sobre la dudosa validez o el carácter reductivo de la tesis sostenida por la doctrina nacionalista acerca del vínculo originario entre sentencia del Estatuto y proceso independentista. Un repaso somero a los hechos nos puede ilustrar: tras la sentencia citada, el Gobierno tripartito catalán (el Partit dels Socialistes de Catalunya [PSC], de izquierdas; Esquerra Republicana de Catalunya [ERC], partido independentista; e Iniciativa per Catalunya Verds [IC-V], herederos del Partido Socialista Unificado de Cataluña [PSUC] o comunistas catalanes), con su presidente, el socialista José Montilla, al frente se suman a una manifestación en Barcelona contra la sentencia del Tribunal Constitucional con el lema "Somos una nación. Nosotros decidimos" (10 de julio de 2010). El Gobierno catalán convoca a elecciones al Parlamento catalán (28 de noviembre de 2010), que gana la federación Convergència i Unió, que había gobernado Cataluña desde 1980 hasta 2003, liderada esta vez por Artur Mas, hombre de confianza del anterior presidente Jordi Pujol. En ausencia de mayoría parlamentaria, el nuevo Gobierno se apoya en los diputados del PP (el mismo partido que había impugnado el Estatuto). En este periodo, las encuestas no muestran un crecimiento notable en el apoyo al independentismo en la opinión pública catalana (en 2010 estaba en 24,3-25,2\% -junio y octubre, tras la sentencia-; en 2011 alcanzaba el 30,3\% -en diciembre- y en 2012 estaba en $34 \%$-en junio- y en 44,3\% -en octubre-, tras la manifestación). ${ }^{3}$ Tampoco las manifestaciones del 11 de septiembre de 2010 y 2011, festividad nacional catalana, fueron muy concurridas. El salto se producirá en la de 2012. Mientras, el nuevo Gobierno catalán tenía que lidiar con la crisis económico-financiera que se había iniciado en 2008, cuyos efectos eran ya muy graves en áreas de competencia autonómica: sanidad, educación, asistencia social o función pública. Ello llevó a enfrentamientos de colectivos sociales con el Gobierno catalán, identificado como el Gobierno de los "recortes" en prestaciones sociales. El momento álgido lo constituyó el bloqueo de la sede del Parlamento catalán que obligó al presidente Mas a salir de allí en helicóptero (17 de junio de 2011).

A esta crisis financiera se añade la crisis institucional derivada de la afloración de varios casos de corrupción política en los principales partidos españoles, que en Cataluña afectaban directamente al partido gobernante (Convergència)

3 Se ha seguido la evolución en los barómetros de opinión pública del Centre d'Estudis d'Opinió (CEO), Generalitat de Cataluña. 
y al expresidente Pujol y su familia (el cual no lo reconoció públicamente hasta enero de 2014).

Ante esta situación, el Gobierno catalán y su entorno mediático emprendieron con fuerza la campaña de culpabilizar a España de las consecuencias de la crisis: una insuficiente financiación de Cataluña impedía hacer frente a la crisis, de ahí el "España nos roba", de resonancias en la Lega Nord (Roma latrona), y la demanda de un "pacto fiscal" con el Estado, similar al régimen de concierto económico del País Vasco y Navarra. Durante aquel periodo, se instaló el debate de las balanzas fiscales, en el que se advertía que Cataluña aportaba más que recibía a la solidaridad interterritorial. Por ello, se transmite la consigna "mejor solos" para lidiar con la crisis (más recursos, mejor gestión). Estas demandas ponen el énfasis en los aspectos socioeconómicos en lugar de la identidad cultural o lingüística, como había sido habitual en el nacionalismo catalán a lo largo del siglo XX. Ello supondrá ampliar las bases sociales independentistas, en un momento de grave crisis económica, más allá de sus caladeros habituales y extenderlos a parte de la población castellano-hablante o de los hijos de la inmigración interior de la década de 1950 y 1960, básicamente radicada en el cinturón industrial de Barcelona. Frente a esta creciente tensión, el nuevo Gobierno del Estado, presidido por Mariano Rajoy (PP), de forma inhábil se limitó a alegar la gravedad de la crisis económica de aquel momento para negarse siquiera a discutir sobre la reivindicación de mayores recursos y mayor autonomía financiera con el Gobierno catalán (otoño de 2012), sin tomarse en serio el desafío independentista. Confiaba en que, superada la crisis, la situación volvería a la normalidad.

Sin negar la relevancia de la sentencia en la movilización del mundo independentista, lo determinante es que supo catalizar un amplio descontento social en la población catalana, desviando, de este modo, la atención en la propia responsabilidad en la gestión de la crisis. La causa principal de dicho descontento, a nuestro juicio, eran las crisis económica y política, las cuales contribuyeron decisivamente a la extensión de la crisis secesionista. De este modo, el proceso independentista supone la respuesta populista que se da en Cataluña a dichas crisis. Las graves crisis de fines de la década de 2000 propiciaron el auge de populismos de distinto tipo en muchos países occidentales (Ginsburg y Aziz, 2018): de izquierdas en España (Podemos como fruto del 15-M y el "no nos representan") o en Grecia, o de derechas en varios países europeos (antieuropeismo y antiinmigración), o de más difícil clasificación: Movimento 5 Stelle en Italia. Con caracteres originales, el proceso catalán comparte rasgos 
fundamentales con los demás populismos mencionados: adopta posiciones en las que prima la emoción, antiinstitucionales y particularmente contra los órganos judiciales (el Tribunal Constitucional); provoca el surgimiento de liderazgos fuertes que lo impulsan (Mas), utilizando toda la estructura del poder de la Generalitat y de los medios de comunicación controlados a su servicio; identifica a un enemigo externo al que culpabilizar de la crisis (España); invoca la expresión directa de la voluntad del pueblo frente a los instrumentos de democracia representativa (el referéndum) y, ante su imposibilidad jurídica, reviste las elecciones al Parlamento catalán de carácter plebiscitario (en 2012 a favor o no de la consulta; en 2015, de la independencia). En definitiva, promueve una concepción de la democracia radical o revolucionaria frente a la democracia constitucional, que reduce la democracia a la expresión de la voluntad de la mayoría expresada en un referéndum, a la vez que rompe la vinculación entre democracia y Estado de derecho, con menoscabo de las resoluciones judiciales (Closa, 2020). Y esto se hace impulsado desde las mismas instituciones del Gobierno catalán.

Si convenimos en que la gran manifestación en Barcelona del 11 de septiembre de 2012 significa el inicio del proceso, ello no supone que este salga de la nada. Se han expresado opiniones de un lado y de otro y se han aportado datos sobre lo que son antecedentes que tienen una influencia en el estallido de la crisis posterior. Así, por un lado, se ha alegado el uso del autogobierno desde la década de 1980, particularmente los medios de comunicación de la Generalitat o la escuela, para ir generando un sentimiento público de afirmación de lo catalán en contraposición a lo español, o de una identidad nacional catalana distinta de la española (historia, cultura, lengua). De 1988 data el conocido "Plan 2000”, elaborado por el presidente Pujol y no conocido hasta años más tarde, con el propósito de "nacionalizar" Cataluña. Por otro lado, ya durante 2009 y 2010 (pendiente la sentencia del Tribunal Constitucional), en municipios catalanes -sobre todo del interior- se habían celebrado consultas en favor de la independencia (Arenys de Munt fue el primero, el 13 de septiembre de 2009), aunque no tuvieran lugar o fracasaran en entornos urbanos mayores. Todo ello preparó el terreno para el estallido independentista en septiembre de 2012.

\section{Desarrollo: etapas del proceso}

Podemos distinguir dos etapas sucesivas desde que el debate independentista se convirtió en prioritario para el Gobierno nacionalista catalán: una soberanista y otra claramente independentista. Se distinguen ambas etapas por la utiliza- 
ción de categorías distintas en la formulación de las reivindicaciones. En las dos se sigue el mismo modus operandi: aprobación por el Parlamento de resoluciones (no vinculantes, ejercicio de la función de impulso político o declarativa), se aprueban normas jurídicas -leyes y decretos de desarrollo- para hacer efectivas tales resoluciones y el Gobierno del Estado impugna unas y otras ante el Tribunal Constitucional: las resoluciones mediante el procedimiento del artículo 161.2 de la CE, las leyes mediante el recurso de inconstitucionalidad. Mientras en la primera fase hay un intento de salvar las formas o de aparentar que no se desea la ruptura, para lo que se busca una interpretación constitucional que dé cobertura a lo que se defiende, en la segunda se olvidan las sutilezas jurídicas y se opta por la fuerza de los hechos. No está claro si existe un plan de escalada independentista desde el inicio que solo se irá desvelando poco a poco, a medida que la ciudadanía vaya asumiéndolo y que el Gobierno del Estado se cierre a negociar, o si más bien la escalada se va improvisando sobre la marcha, a medida que los radicales van dominando la escena, como suele ocurrir en los procesos revolucionarios.

\subsection{Primera etapa: soberanista (2012-2015)}

La primera etapa abarca desde la manifestación del 11 de septiembre de 2012 hasta las elecciones del 27 de septiembre de 2015. El proceso estuvo marcado por la reclamación del "derecho a decidir" la secesión. Esto es, no se trata estrictamente de que el pueblo catalán decida la secesión en un referéndum vinculante, sino más bien de una apelación al pueblo a través de algún tipo de consulta popular para recabar su opinión, a los efectos de impulsar y legitimar la actuación posterior de las instituciones públicas catalanas tendente a negociar o a lograr la secesión. En esta primera etapa se hablaba más de consulta que de referéndum estrictamente. El "derecho a decidir" es una construcción político-jurídica distinta del derecho de autodeterminación, ${ }^{4}$ que se crea precisamente ante la dificultad de encajar el caso catalán dentro de los supuestos de autodeterminación reconocidos en el derecho internacional. Tiene su origen en el País Vasco en la década anterior (segundo Plan Ibarretxe, 2008) y apela al principio democrático y al derecho fundamental de participación política, ambos establecidos en la Constitución española. ${ }^{5}$

4 Véase Pons Rafols (2015).

5 Sobre la creación y justificación del derecho a decidir, véase Barceló i Serramalera et al. (2015). 
Tras la manifestación del 11 de septiembre, se convocaron a elecciones al Parlamento catalán, anticipadas, para el 25 de noviembre de 2012. Antes, a modo de despedida, el Parlamento aprobó la resolución 472/IX, de 27 de septiembre, en la que se constató la voluntad del pueblo de Cataluña de decidir su futuro colectivo libre y democráticamente a través de una consulta. En las elecciones, la federación gobernante Convergència i Unió (CiU), liderada por el entonces presidente de la Generalitat, Artur Mas, gana aunque baja de 62 a 50 escaños, con un programa soberanista y con una campaña publicitaria muy personalista, en la que él aparece como quien encarna "la voluntad de un pueblo”. Este retroceso electoral se compensó con el apoyo de las demás fuerzas nacionalistas, ERC en particular. El primer pleno ordinario del Parlamento aprobó una resolución que se refiere al inicio del "proceso para hacer efectivo el ejercicio del derecho a decidir" (no la independencia), en la que se declara "por razones de legitimidad democrática" al pueblo de Cataluña como "sujeto político y jurídico soberano” (Resolución 5/X, de 23 de enero de 2013). El Gobierno del Estado recurrió dicha resolución ante el Tribunal Constitucional, el cual resolvió la impugnación en la sentencia 42/2014. Dicha sentencia supone el mayor intento de acercamiento de posiciones por parte del Alto Tribunal, al declarar inconstitucional el carácter soberano del pueblo catalán al tiempo que admite como "aspiración política" legítima -no un derecho- la defensa del derecho a decidir. Para ello, introduce un matiz relevante a su doctrina anterior: ${ }^{6}$ la distinción entre los actos preparatorios y la reforma constitucional misma. Así, se indica:

El planteamiento de concepciones que pretendan modificar el fundamento mismo del orden constitucional tiene cabida en nuestro ordenamiento, siempre que no se prepare o defienda a través de una actividad que vulnere los principios democráticos, los derechos fundamentales o el resto de los mandatos constitucionales, y el intento de su consecución efectiva se realice en el marco de los procedimientos

6 Especialmente la sentencia 103/2008 (en relación con el segundo Plan Ibarretxe o propuesta de consulta popular en el País Vasco). En ella, el Tribunal sienta las bases de su posición sobre los referéndums o consultas de autodeterminación: sobre cuestiones de orden constitucional han de decidir todos los españoles a través del procedimiento de reforma constitucional. A diferencia de otros ordenamientos constitucionales, toda la Constitución española es reformable, inclusive la unidad e indivisibilidad del Estado, sin que haya pues límites a la reforma o cláusulas de intangibilidad expresas o tácitas. Ahora bien, dicha reforma ha de hacerse siguiendo el procedimiento de revisión establecido en ella, esto es, el procedimiento más agravado previsto para la reforma total o de las partes fundamentales (artículo $168 \mathrm{CE}$ ). En consecuencia, no cabe que el legislador ordinario, estatal o territorial, pueda decidir la independencia de una Comunidad autónoma o fijar el procedimiento para ello. 
de reforma de la Constitución, pues el respeto a esos procedimientos es, siempre y en todo caso, inexcusable. (FJ 4) ${ }^{7}$

De este modo, el Tribunal da a entender que antes de iniciar el procedimiento de reforma propiamente dicho cabría la realización de determinados "actos". Pero no aclara qué tipo de actos son admisibles en la fase preparatoria, solo que deberán respetar los principios y demás preceptos constitucionales. Al final, de acuerdo con la sentencia, el derecho a decidir queda acotado no como un derecho a negociar (como en Canadá, fruto del conocido Dictamen del Tribunal Supremo de 1998 sobre la secesión de Quebec), sino como un derecho a deliberar o debatir en las Cortes Generales las iniciativas de reforma que presenten los parlamentos territoriales, sin que puedan aquellas rechazar de plano la propuesta. Ello se vincula, en la sentencia, con la reiteración de su doctrina acerca de la inexistencia de límites materiales a la Constitución y de que los partidos puedan sostener ideas contrarias a la Constitución, sin ser disueltos por ello. 8

Para ejercer el derecho a decidir, los asesores del Gobierno catalán -constituidos en el denominado Consejo Asesor para la Transición Nacional (Decreto 113/2013, de 12 de febrero)- habían propuesto varias vías a seguir para conseguir la consulta a la ciudadanía. También la doctrina constitucionalista debatió en aquel momento sobre la posibilidad de organizar un referéndum. A partir de la inexistencia expresa en la Norma Fundamental española de una cláusula sobre la secesión, entendida como derecho a la autodeterminación o como procedimiento para poder alcanzarla, ni de un referéndum como instrumento para llevarla a cabo, buscaron una interpretación -a nuestro juicio, muy forzada- de los preceptos constitucionales que lo hiciera posible. ${ }^{9}$ De las vías

7 Cursiva añadida.

8 Véase una crítica a esta construcción jurisprudencial en De Miguel Bárcena (2019).

9 Aunque la doctrina constitucionalista mayoritariamente rechazó la posibilidad de un referéndum de secesión, salvo su inclusión previa en una reforma constitucional, algunos autores trataron de buscar en la Constitución vigente algún resquicio que posibilitara la convocatoria de un referéndum. Así, Aguado Renedo (2013) abría la puerta a un referéndum consultivo sobre la independencia que vendría a satisfacer una razón "puramente práctica", como es poner en marcha o no el procedimiento de reforma constitucional del artículo $168 \mathrm{CE}$. De este modo, habría un referéndum para "testar" o tratar de verificar la voluntad de reforma de los catalanes, y un referéndum al final del procedimiento de reforma para el conjunto del pueblo español. En este sentido, se menciona la posible -aunque forzada- aplicación del referéndum consultivo del artículo $92 \mathrm{CE}$, el cual -sobre una "decisión política de especial trascendencia" - es de carácter consultivo y tiene que ser propuesto por el presidente del Gobierno español con la aprobación de la mayoría absoluta del Congreso de los Diputados. Para ello, habría que salvar un obstáculo literal del artículo 92 CE: se convocaría 
propuestas por los asesores del Gobierno catalán se intentaron seguir dos por parte de las instituciones catalanas, ambas sin éxito: ${ }^{10}$ la primera, la delegación por el Congreso al Parlamento catalán de la competencia sobre la autorización de la convocatoria del referéndum (artículo 149.1.32, CE); el Congreso rechazó en abril de 2014 la proposición de ley del Parlamento catalán al respecto. Y la segunda, la celebración de una denominada "consulta popular no referendaria” en lugar del referéndum; el Tribunal Constitucional la declaró inconstitucional (sentencias 31 y 32/2015). En su lugar se llevó a cabo un "proceso de participación ciudadana" el 9 de noviembre de 2014, equivalente a la consulta y al referéndum -las mismas preguntas a responder a través del voto- cuyo resultado no tuvo el efecto clarificador deseado. ${ }^{11}$

Ambas vías intentadas tienen en común la organización por el legislador catalán de una "consulta" que debería servir para "testar" la voluntad de los catalanes y abrir la puerta a una negociación bilateral sobre la secesión o a una reforma constitucional que la permitiera, aunque el desenlace de toda esta compleja construcción jurídica distaba de estar clara. ${ }^{12}$ De entrada, se trataba

a la fracción del cuerpo electoral formado por la población del territorio catalán y no a "todos los ciudadanos", hasta ahora entendido como los ciudadanos españoles. Para Rubio Llorente (2012; 2013), la convocatoria de un referéndum en Cataluña sería la opción última en caso de no existir otra vía de encauzar la situación. Se trataría de un referéndum no previsto en la Constitución, regulado específicamente para el caso excepcional.

10 En el primer informe del Consejo Asesor para la Transición Nacional se establecen cinco vías para conseguir el ejercicio del derecho a decidir. Además de la reforma constitucional, contempla las siguientes "vías" jurídicas: el referéndum consultivo del artículo 92 CE regulado por la Ley orgánica 2/1980 de referendos y que debería convocar el Congreso de los Diputados a propuesta del presidente del Gobierno del Estado; la delegación por las Cortes Generales al Gobierno catalán de la competencia sobre autorización de la convocatoria para la celebración de referendos (artículo 149.1.32 CE); el referéndum, también consultivo, previsto en la Ley catalana 4/2010 de consultas populares por vía de referéndum; y la denominada "consulta popular no referendaria", que sería aprobada posteriormente por el Parlamento catalán en la Ley 10/2014, de 27 de septiembre. Ver Consell Assessor per a la Transició Nacional (2014).

11 Se formularon dos preguntas: si se quería que Cataluña fuese un Estado y si se quería que fuese un Estado independiente. Según los datos oficiales, participaron 2344828 votantes, esto es un 33\% de los que tenían derecho a hacerlo. Votó sí a la independencia el 80,9\%; a favor de que Cataluña se convirtiera en un Estado (sin aclarar a qué tipo de Estado se refiere), el 10,02\%; y en contra de ambas opciones, por tanto, por el status quo, un 4,49\%.

12 Los intentos de hallar una vía que permitiera una celebración de una consulta referendaria de conformidad, o al menos no contraria al ordenamiento constitucional español, abocan a una situación a considerar: un resultado del referéndum favorable a la secesión ¿a qué llevaría? Para algunos, a la apertura del procedimiento de reforma, como se ha visto. Se trata de un itinerario complejo y de desenlace incierto, que escapa del unilateralismo catalán en la decisión. Más bien parece que en ese momento se produciría una ruptura con el ordenamiento español y dicho resultado se convertiría en un hecho revolucionario, el cual daría paso a la política en su sentido más descarnado. Ver Caste- 
de sortear el régimen constitucional del referéndum y sus límites, en particular la preceptiva autorización de su convocatoria por el Gobierno del Estado (artículo 149.1.32, CE). Por ello, la segunda vía ensayada supone la creación de unas llamadas "consultas populares no referendarias", como tertium genus entre el referéndum propiamente dicho y las consultas populares clásicas, esto es, modalidades participativas sin ejercicio del voto. Es lo que contemplaba la Ley 10/2014, que fue anulada en este punto por el Tribunal Constitucional en la sentencia 31/2015. Para el Alto Tribunal, dichas consultas en realidad son referendos encubiertos, pues apelan al cuerpo electoral a través del voto. El Tribunal aplica la doctrina establecida en la sentencia 103/2008, en la que se fijan los requisitos para que pueda hablarse de referéndum, que en la sustancia se aplicarían a las nuevas consultas no referendarias: llamada al cuerpo electoral (aunque en la consulta se añadan a este -formado por los ciudadanos españoles mayores de edad residentes en municipios catalanes- otros participantes, como los mayores de entre 16 y 18 años o los extranjeros residentes); que se siga el procedimiento previsto para el régimen electoral; y que cumpla con las garantías judiciales establecidas en la legislación electoral. ${ }^{13}$

Sin embargo, merece destacarse que desde el inicio del proceso independentista en 2012, y a pesar de ser una de las "vías" jurídicas contempladas por los asesores del Gobierno catalán para ejercer el derecho a decidir, el Parlamento catalán nunca ha ejercido la iniciativa de reforma constitucional (de acuerdo con los artículos 166 y 87.2, CE) para proponer la introducción de un referéndum de secesión. ${ }^{14}$

llà Andreu (2014, pp. 829-830). Desde otra perspectiva, Jordi Jaria i Manzano (2015) distingue entre un primer momento de constatación de la voluntad de la ciudadanía catalana, de acuerdo con la Constitución, y un segundo momento de realización de la ruptura constitucional "inevitable".

13 El Tribunal declaraba inconstitucional la ley vasca de 2008 que preveía una consulta popular ad hoc sobre el futuro político del País Vasco. Para el Tribunal, el hecho de que la ley del País Vasco tratara de distinguir dicha "consulta" de un referéndum no permitía soslayar la aplicación del régimen jurídico general de los referendos autonómicos que, de acuerdo con la Constitución, han de contar con la preceptiva autorización del Estado (artículo 149.1.32) y estar previstos expresamente en el Estatuto de autonomía, además de en la Ley orgánica de referéndum. Por tanto, no cabe una competencia implícita de las Comunidades Autónomas sobre referéndum. Se trataba de consideraciones válidas para todo referéndum territorial, no específicamente para uno de secesión.

14 De acuerdo con este, la iniciativa la ostentan, entre otros, los Parlamentos de las Comunidades Autónomas (pero no una fracción del cuerpo electoral, como sí ocurre con la iniciativa legislativa popular para la aprobación de leyes ordinarias). La participación de las Comunidades en cuanto tales concluye con la presentación de la propuesta. A partir de esta, corresponde a las dos Cámaras de las Cortes Generales la aprobación, primero, de los principios básicos de la reforma y, tras unas elecciones generales intermedias, la discusión y aprobación definitiva por $2 / 3$ de los miembros de cada una de las nuevas Cámaras, así como al pueblo español en referéndum la ratificación al final del proceso (artículo $168 \mathrm{CE}$ ). 


\subsection{Segunda etapa: independentista (2015-2017)}

Ante el aumento de la tensión con las instituciones estatales (y la ausencia de una mayoría de Gobierno clara en la Cámara), el presidente de la Generalitat Mas convocó a elecciones anticipadas al Parlamento catalán para el 27 de septiembre de 2015 con un objetivo "plebiscitario", esto es, que los electores catalanes, llamados a elegir a sus representantes, expresaran con su voto su posición ante la independencia. Ante la imposibilidad de celebrar un referéndum, llama la atención la frecuencia en la convocatoria de elecciones al Parlamento catalán desde el inicio de la crisis $(2012,2015,2017)$, lo que muestra tanto la división del mundo independentista y el intento de hallar un aval popular a las medidas adoptadas como la debilidad de los sucesivos Gobiernos.

En esta ocasión, Convergència (tras romper la federación con Unió) y ERC formaron candidaturas conjuntas, con independientes (Junts pel si), que no alcanzaron el $40 \%$ del voto popular y juntos sumaron 62 escaños. Estas elecciones fueron la segunda oportunidad, tras la celebración del proceso participativo indicado (y sin contar las elecciones al Parlamento catalán de 2012, en las que también se suscitó la cuestión por los partidos independentistas), que han tenido los catalanes de manifestar la opinión en las urnas respecto al proceso secesionista y cuyos resultados ponen de manifiesto que no existe una mayoría popular amplia capaz de culminar el proceso independentista con éxito, o, lo que es lo mismo, que la población catalana se halla dividida en torno a la cuestión de la independencia. A pesar de estos resultados, el mundo independentista optó por seguir adelante, es más, por apretar el acelerador de su causa. Se produjo la investidura de Carles Puigdemont como presidente de la Generalitat tras la renuncia de Mas y gracias a la alianza parlamentaria con la Candidatura de Unidad Popular (CUP) (necesaria para alcanzar la mayoría absoluta); se trata de un partido de extrema izquierda que empuja para que el proceso vaya hacia adelante lo más rápido posible y de forma radical.

La segunda etapa, de carácter abiertamente rupturista (2015-2017), se inicia siguiendo el mismo modus operandi que en la etapa anterior: con la aprobación por el nuevo Parlamento que sale de las elecciones del 27 de septiembre de 2015 de una resolución (1/XI, de 9 de noviembre de 2015) en la que se declara el inicio de un nuevo proceso político y constituyente. En ella ya no se habla del derecho a decidir, sino del inicio del proceso "para crear un Estado catalán independiente en forma de República" y la apertura de un "proceso constituyente". Asimismo, se aboga expresamente por desobedecer a las instituciones españolas y, en particular, al Tribunal Constitucional. El Gobierno del Estado 
también impugnó ante el Tribunal Constitucional dicha declaración, que fue anulada en la sentencia 259/2015, la cual llevó a cabo una defensa cerrada de la democracia constitucional y cerró la puerta a la interpretación de la doctrina nacionalista de la sentencia 42/2014, en el sentido de que esta permitiría una consulta popular de tipo prospectivo o anterior al inicio del procedimiento de reforma, esto es, antes de que el Parlamento catalán presentara una propuesta de reforma constitucional al Congreso. ${ }^{15}$ La sentencia recuerda los límites constitucionales a la consulta: no cabe ningún tipo de consulta que afecte a decisiones propias del poder constituyente. De acuerdo con el procedimiento de reforma, la intervención del pueblo español en referéndum tiene lugar al finalizar el procedimiento de forma ratificatoria. ${ }^{16}$

La actividad parlamentaria se centró en avanzar en la causa de la independencia. Así, el Parlamento catalán creó una comisión de estudio cuyas conclusiones abogan por la celebración de un referéndum unilateral y vinculante. El Pleno del Parlamento aprobó la resolución del 6 de octubre de 2016, la cual pone una fecha para su celebración: antes de octubre de 2017 (Resolución 306/ XI). Dicho referéndum habría de versar explícitamente sobre la independencia, ya fuese acordado con el Estado, ya fuese unilateral. Así, pues, en esta nueva etapa -a diferencia de la anterior- se proclama el derecho a la autodeterminación (en lugar del derecho a decidir), y se insta al Gobierno catalán a que celebre un referéndum "vinculante" (ya no se habla de consulta popular) sobre la independencia con una pregunta clara y respuesta binaria -esto es, sí o no- (ya no se dan otras opciones como había sucedido en 2014). Los presupuestos de la Generalitat para 2017, aprobados por el Parlamento catalán en marzo de ese año, consignan una partida para financiar la celebración de dicho referéndum.

Todos esos actos parlamentarios, de distinta naturaleza jurídica, fueron impugnados por el Gobierno del Estado ante el Tribunal Constitucional -también siguiendo el patrón ya visto-, que los suspendió y anuló posteriormente. En relación con las resoluciones parlamentarias citadas, el Tribunal aplicó un instrumento establecido por la reforma de 2015 de la Ley Orgánica del Tribunal Constitucional, el incidente de ejecución de sentencias, por el cual el Tribunal puede adoptar medidas ejecutivas de las sentencias y contra las autoridades que

15 Véase Ridao Martin (2014, pp. 110 y ss.).

16 Un intento nada fácil de armonizar ambos pronunciamientos del Tribunal pasaría por descartar las consultas de tipo referendario en la fase previa a la apertura de la reforma propiamente dicha y admitir otro tipo de instrumentos participativos, como audiencias, encuestas o foros. Véase Castellà Andreu (2016). 
incumplan sus resoluciones. Tales actos aprobados eran contrarios a la sentencia del Tribunal Constitucional 259/2015, por lo que se conminaba a su cumplimiento con amenaza de sanción a los que no lo hiciesen y con la petición al Ministerio Fiscal para que abriera diligencias penales contra los responsables.

Esta fase de escalada concluye con los sucesos de septiembre y octubre de 2017, el momento culminante de la crisis. El Parlamento catalán aprobó las llamadas "leyes de desconexión": la ley de referéndum de autodeterminación (Ley 19/2017, de 6 de septiembre) y la ley de transitoriedad jurídica hacia la república (Ley 20/2017, de 8 de septiembre), una suerte de constitución provisional para regular el tránsito de la situación actual al nuevo Estado y hacerlo, en palabras de sus promotores, "de la ley a la ley" (expresión tomada -impropiamente- de la utilizada por el presidente de las Cortes Generales en 1976, Torcuato Fernández-Miranda, para aludir a la Ley para la Reforma Política de 1977 como "ley puente" entre el ordenamiento franquista y el democrático). Dichas leyes fueron aprobadas por la mayoría independentista siguiendo un procedimiento expedito (discusión en el Pleno en menos de 24 horas, sin paso previo por Comisión y sin dictamen del órgano jurídico consultivo de la Generalitat, como había solicitado la oposición). En su articulado, ambas leyes establecían su superioridad respecto al ordenamiento jurídico de pertenencia (la Constitución y el Estatuto) (artículo 3 de las dos leyes). De acuerdo con las leyes citadas, en caso de ganar la opción independentista en el referéndum vinculante (por mayoría simple, sin exigencia de quorums especiales), se proclamaría la independencia y se abriría un proceso participativo que desembocaría en la creación de una Asamblea Constituyente -tras la celebración de una nuevas elecciones-, la cual debía aprobar la constitución de la nueva república catalana. A través de un segundo referéndum, el pueblo catalán se pronunciaría sobre la nueva constitución catalana. El Tribunal Constitucional, tras suspender inmediatamente la aplicación de dichas leyes, las declaró inconstitucionales en las sentencias 114 y 120/2017, alegando vicios de procedimiento y de contenido. Concretamente, no habían respetado en su tramitación los derechos de las minorías parlamentarias y sus contenidos se situaban en una posición de total ajenidad al ordenamiento español, contraviniendo radicalmente los principios básicos de la Constitución: soberanía del pueblo español, superioridad de la Constitución y unidad de España (artículos 1.2, 9.1 y 2, CE).

Sin embargo, el 1 de octubre de 2017 tuvo lugar el referéndum inconstitucional, en una jornada con incidentes con la policía y en la que no se observa- 
ron las garantías propias de una votación. ${ }^{17}$ Contó con la supervisión de una Sindicatura Electoral, órgano creado ex profeso por la mayoría independentista del Parlamento catalán, con la presencia de miembros elegidos por este sector sin participación de la oposición. El referéndum abría la semana más decisiva de todo el proceso y que decantó su fracaso: el 3 de octubre, el Rey de España intervino públicamente en defensa del ordenamiento constitucional y la unidad de la nación, la Unión Europea y los Gobiernos de los Estados miembros unánimemente se posicionaron claramente en contra, miles de ahorristas y empresas catalanes trasladaron sus fondos o domicilios fiscales fuera de Cataluña y la semana culminó el 8 de septiembre con una manifestación masiva en Barcelona contra la independencia. Pese a todo esto, el Parlamento decidió seguir adelante con su plan: aprobó la declaración de independencia, que se suspendió al instante (27 de octubre), y no obtuvo ningún reconocimiento internacional. Las instituciones del Estado respondieron el mismo día con la aprobación por el Pleno del Senado, a propuesta del Gobierno, del acuerdo con las medidas coercitivas del artículo 155 de la CE (con los votos de PP, PSOE, Ciudadanos [Cs] y algunos grupos regionalistas). Ello suponía cesar al Gobierno catalán, disolver el Parlamento -convocando a elecciones autonómicas para el 21 de diciembre- y asumir las competencias directamente por parte del Gobierno del Estado. También el Tribunal Constitucional se pronunció de forma unánime (igual que en el resto de las sentencias sobre el proceso independentista), estimando la constitucionalidad de las medidas excepcionales, impugnadas por los diputados de Podemos en el Congreso y por el Parlamento catalán (Sentencia 89 y 90/2019).

\section{Situación presente: distensión y división en el independentismo (2018-2020)}

La celebración de las elecciones al Parlamento del 21 de diciembre de 2017, a las que, esta vez, las fuerzas independentistas concurrieron por separado -aunque juntas revalidaron la mayoría absoluta de escaños (Junts per Catalunya, ERC, CUP: 70 escaños, frente a los 72 de 2015)-- ${ }^{18}$ dio lugar a un nuevo perio-

17 Los resultados fueron, según sus convocantes: 43\% de participación con un $90 \%$ de "si”", un 7,83\% de "no" y un $1,98 \%$ de votos en blanco.

18 Hay que advertir que el independentismo logra mayorías parlamentarias sin haber obtenido hasta ahora una mayoría en votos populares. Ello se debe a la distribución de escaños en las cuatro provincias catalanas, establecida en el Estatuto catalán de 1979 y que ha permanecido inalterada hasta ahora, pese a los cambios demográficos. Dicha distribución otorga sobrerrepresentación a las circunscripciones menos pobladas, lo que se traduce en desigualdad en el voto (el de Lleida vale el doble que el de Barcelona). Es en las circunscripciones menos pobladas donde las fuerzas indepen- 
do político y jurídico -en el que aún estamos-, caracterizado, en el orden político, básicamente por la distensión y la apertura de diálogo entre los Gobiernos central y catalán, tras el cese de las medidas excepcionales del artículo 155 de la CE y la formación de un nuevo Gobierno en Madrid a lo largo de 2018. En este periodo, el conflicto constitucional ha disminuido sustancialmente y el juicio penal en el Tribunal Supremo contra los máximos responsables de la organización del referéndum, así como su condena, han ocupado una atención especial. Frente a las demandas de referéndum de secesión del periodo previo, ahora han sobresalido las peticiones de libertad para los presos y de restablecimiento de la autonomía (durante la vigencia de las medidas excepcionales del artículo 155, CE). Curiosamente, ello evoca las reivindicaciones de la década de 1970: Llibertat, amnistia i Estatut d'autonomia.

Sobresalen los siguientes factores políticos en este último periodo: en primer lugar, la dificultad para formar Gobierno en Cataluña, lo que no se consiguió hasta el 17 de mayo de 2018 con la elección de Joaquim Torra (Puigdemont había huido a Bruselas tras aprobarse la declaración de independencia y no pudo ser investido, aunque desde allí ha seguido como líder del "legitimismo" independentista más radical). Durante esos meses sin constituir Gobierno, se mantuvo el control de la Administración autonómica por la estatal, de acuerdo con las medidas coercitivas del artículo 155 de la CE. En segundo lugar, un cambio de escenario político en Madrid a partir de la moción de censura contra Mariano Rajoy, que llevó al líder socialista Pedro Sánchez a la presidencia del Gobierno, con los votos de todos los representantes independentistas catalanes en el Congreso (1 de junio de 2018), y que se confirmó tras las elecciones generales de abril de 2019 con victoria socialista, aunque sin poder formar Gobierno, hasta el punto de obligar a repetir los comicios en noviembre de 2019: el nuevo Gobierno es el primero de coalición en España, entre el PSOE y Unidas-Podemos, y con apoyo parlamentario, entre otros, de ERC. Sánchez prometió, a cambio de este apoyo, una mesa bilateral de negociación con la Generalitat, que llegó a reunirse al máximo nivel el 6 de febrero de 2020 en Barcelona, siguiendo los pasos del primer encuentro en el Palacio de Pedralbes de 20 de diciembre de 2018. Sin embargo, la pandemia originada por el COVID-19 trastocó completamente las prioridades y esta vía negociadora ha quedado suspendida por el momento. Por último, mientras tanto, han ido en aumento las disputas en el interior del sector independentista entre radicales 
y pragmáticos, además de producirse varias escisiones en el Partido Demócrata Europeo Catalán (PDeCAT)-Junts per Catalunya (herederos de Convergència). Dichas tensiones han provocado que el Parlamento catalán apenas legisle (la ley de presupuestos de 2020 se aprobó en abril y no se habían aprobado los presupuestos desde 2017). A ello se le suma el posible cese del Presidente catalán por el Tribunal Supremo por desobediencia a las autoridades judiciales (otoño de 2020), lo que desembocará previsiblemente en otras elecciones anticipadas en Cataluña (las cuartas desde 2012, cuando una legislatura ordinaria dura 4 años).

Este nuevo escenario político se completa con el judicial. El Tribunal Supremo juzgó y condenó a elevadas penas de prisión por delito de sedición (en lugar del más grave de rebelión, solicitada por el Ministerio Fiscal y la acusación particular ejercida por Vox) a los responsables del Gobierno catalán en la organización del referéndum ilegal que no habían huido al extranjero (sentencia 459/2019, de 14 de octubre). A los pocos meses, se estaban aplicando ya beneficios penitenciarios a los condenados, que les permitieron salir de prisión. Asimismo, el conflicto jurídico se ha centrado, en este periodo, en la reclamación ante distintos tribunales nacionales y europeos de parlamentarios electos huidos de la justicia que no han podido tomar posesión de sus cargos representativos (siendo reemplazados por el siguiente en la correspondiente candidatura), que alegan la vulneración de su derecho de acceso a cargos públicos (artículo 23.2, CE), además de otras denuncias de violación de derechos procesales durante el juicio.

Mientras tanto, según los datos de las distintas encuestas de opinión pública, el voto pro independencia está en el punto más bajo desde 2012 (42\% de los catalanes, según el último sondeo del CEO -instituto oficial de la Generalitat- del 31 de julio de 2020, frente al 50,5\% de contrarios, en respuesta a la pregunta binaria por la independencia; la más alta posición pro independencia se alcanzó en octubre 2017, coincidiendo con el momento álgido de la crisis: 48,7\%, y en abril de 2018: 48\%). Al mismo tiempo, al preguntar por las distintas opciones posibles de organización del Estado, un 33,9\% de los catalanes encuestados se muestra partidario de la creación de un Estado independiente (frente al 35,5\% de la encuesta previa del CEO), la posición más baja desde 2014; un 29,6\%, de que Cataluña sea una Comunidad Autónoma dentro del actual Estado de las autonomías (frente al 26,8\% anterior, la más alta desde 2014), a lo que se suma un 6,8\% que opta por Cataluña como región de España y un 22,9\% a favor de un Estado en un Estado federal (frente al 23,9\% anterior). ${ }^{19}$

19 Véase: http://upceo.ceo.gencat.cat/wsceop/7688/Dossier\%20de\%20premsa\%20-974.pdf. 
A la vista de los hechos y de los datos citados, esta nueva etapa del proceso independentista se ha caracterizado, primero, por una paralización efectiva de las iniciativas independentistas en el Parlamento catalán que puedan suponer consecuencias penales a sus proponentes. La tensión dialéctica ha permanecido en el sector independentista más radical, aunque sus dirigentes se han cuidado de no traspasar, en términos generales, los límites de la legalidad, sabedores de las consecuencias de orden penal que ello les acarrearía. Ahora, sus críticas se centran en el trato a los presos, visto como una herida. Segundo, una parte del independentismo, fundamentalmente los partidos (ERC, después incluso el PDeCAT, tras romper con Junts per Catalunya), han optado por abrirse a negociaciones, o al menos a un diálogo político con el nuevo Gobierno de Madrid. Frente a ello, el sector más radical -vinculado a Puigdemont-Torra- sigue en la confrontación con el Estado y en canalizar sus demandas independentistas a través de un movimiento (Junts per Catalunya) de connotaciones populistas. El Gobierno de Sánchez aceptó una negociación bilateral -entre Gobiernos- y prometió aparcar la judicialización del conflicto. Se están por ver los contenidos y los resultados de la negociación cuando esta se reanude, aunque por el momento la tensión social ha bajado en términos generales (tras unas semanas de gran violencia en las calles de Barcelona, en octubre de 2019, coincidiendo con la publicación de la sentencia del Tribunal Supremo), en parte porque otros asuntos son priorizados por la ciudadanía (crisis sanitaria, crisis económica derivada de la primera). Mientras tanto, el Gobierno de la Generalitat sigue reduciendo al máximo su presencia en foros multilaterales intergubernamentales (inasistencia a la XXI Conferencia de Presidentes Autonómicos el 31 de julio; aunque había asistido a las reuniones telemáticas para la gestión de la pandemia). En cualquier caso, parece evidente que cada vez se deja más de lado la vía independentista unilateral y contraria al ordenamiento jurídico.

Por último, el independentismo (y Podemos) ha decidido situar en este último periodo en su punto de mira a la Corona, sabedores de que ello supone atacar los cimientos del actual sistema constitucional y su origen, la transición política a la democracia. De ahí que el Parlamento catalán intentara la creación de una comisión de investigación sobre la Monarquía (Resolución 298/ XII), que el Tribunal Constitucional declaró inconstitucional en la sentencia 111/2019, y ha culminado esta tensión institucional con la aprobación, con los votos independentistas, de unas resoluciones contra la Monarquía, el actual Rey y el anterior y proclamando que Cataluña es republicana (en particular, la Resolución 905/XII, de 7 de agosto de 2020, aunque la presidencia de la 
Cámara no se atrevió a publicarlas enteras en el Boletín Oficial del Parlamento para no desobedecer al Tribunal Constitucional e incurrir en responsabilidades penales).

\section{Perspectivas de futuro: salidas constitucionales}

Se han apuntado varias opciones políticas y constitucionales para salir de la crisis en la que nos hallamos. Hasta que no se clarifique el escenario político catalán, con la celebración de elecciones, no sabremos cuál es la mayoría de Gobierno que se forma y qué pasos se dan para afrontar la situación: continuidad de la mayoría independentista o apertura a otras coaliciones, como una de izquierdas (nuevo tripartito). También habrá que ver los pasos que dé el Gobierno de Sánchez, absorto en la gestión de la crisis originada por el COVID-19 y sus gravísimos efectos en la economía: si podrá mantener los votos de la investidura -con Podemos e incluyendo a los independentistas de ERC- o bien se apoyará en otros grupos parlamentarios -como Ciudadanos o hasta el PP-, sobre todo de cara a la aprobación de la ley de presupuestos y al cumplimiento de las exigencias de Bruselas.

En cualquier caso, y al margen de las circunstancias políticas del momento, el diagnóstico que se haga de las causas que han llevado a los sucesos que se han sintetizado en las páginas anteriores influirá en las propuestas de salida política y constitucional que se formulen. Si se considera que lo sucedido tiene que ver con los déficits del autogobierno (competencial, financiero, simbólico) y el no reconocimiento de una posición singular de Cataluña en el Estado y del derecho a decidir, las salidas a explorar tenderán a corregir esta deriva, en parte originaria del sistema constitucional, en parte decantada por el desarrollo del Estado autonómico. En este caso, las reformas constitucionales pasarán a primer plano. Si, en cambio, se considera que la crisis procede más bien de otro tipo de problemas que se han acentuado en un entorno populista -sentimientos de agravio avivados por políticos desde el poder y sus antenas mediáticas en momentos álgidos de una grave crisis multifacética-, las respuestas serán más complejas de formular y sistematizar por su heterogeneidad, sobre todo porque trascienden las soluciones constitucionales y el plano jurídico, en general, e inciden más bien en el ámbito del cambio político y, sobre todo, en lo prepolítico: la recuperación de la amistad cívica entre catalanes y entre Cataluña y el resto de España, así como de los valores de la democracia constitucional y pluralista. De todos modos, en este momento, cualquier aportación propositiva no 
puede ser sino provisional, a la espera de una mayor clarificación en el plano político. Por ello, nos limitaremos a recordar las opciones y escenarios que se han apuntado con una valoración sucinta de ellas.

Existen divergencias y convergencias sobre la forma de afrontar la crisis habida y las propuestas a considerar tanto en el lado independentista como en el constitucionalista. En el independentista, la coincidencia está en el objetivo final, que hasta ahora ha sido un aglutinante muy poderoso frente a las discrepancias ideológicas de los grupos (desde la extrema izquierda de la CUP hasta los antes moderados de PDeCAT): la independencia de Cataluña, y también en trabajar para alcanzarlo desde las instituciones de la Generalitat y locales en las que se gobierna. Ello se traduce en una ocupación monopolista del espacio público e institucional en Cataluña: banderas y símbolos independentistas en edificios y lugares públicos y discurso independentista dominante en los medios de comunicación de la Generalitat. Al mismo tiempo, hay una discrepancia cada vez más visible entre radicales y posibilistas. Mientras los primeros rechazan cualquier negociación y son partidarios de mantener la escalada de tensión con el Estado (Puigdemont y Torra), los segundos (ERC y ahora PDeCAT) asumen la inviabilidad de la independencia en este momento al no darse una mayoría social suficiente en la población catalana y carecer de apoyo o cobertura internacional y de la Unión Europea (UE) a la creación de un Estado catalán independiente (que quedaría fuera de la UE). Se recupera el eslogan pujolista de las décadas de 1980 y 1990: "Ahora paciencia, mañana independencia". Mientras tanto, se abren, no sin dificultades y contratiempos (la presión de los radicales), a la negociación con el Gobierno de izquierdas de Madrid: presupuestos, amnistía a los condenados, relación bilateral con el Gobierno del Estado. En definitiva, una versión puesta al día del viejo paradigma de actuación de $\mathrm{CiU}$ en el Congreso, en Madrid, aunque con una presión en contra muy superior y con una hegemonía institucional sin precedentes que lucha por controlar instancias sociales relevantes (cámaras de comercio, universidades, Barça). Por esta vía de -en términos de Ortega y Gasset- "conllevancia" provisional (sin saber lo que deparará el futuro) abogan sectores económicos y mediáticos influyentes en Cataluña.

En cambio, en el sector constitucionalista, se ha constatado que el acuerdo entre el PSOE, PP y Ciudadanos solo se produjo en el momento álgido de la crisis -septiembre y octubre de 2020 - y únicamente es viable en circunstancias muy especiales y cuando lo fundamental -la unidad de España y el respeto al orden constitucional- está en grave peligro. Alejado el peligro inminente, la 
política vuelve a sus cauces ordinarios de controversia izquierda/derecha, que es la dominante en la política española, en la que la izquierda, para gobernar, necesita en el Congreso los votos independentistas y nacionalistas y prefiere este pacto a ensayar fórmulas de gran coalición con el PP. La entrada de Podemos en el Gobierno ha contribuido a aumentar la radicalización de este, a la vez que se ha constituido en interlocutor o puente entre los socialistas y los independentistas. En lo que ahora interesa, hay divergencias muy profundas entre socialistas y los partidos de centro y derecha (PP, Cs y Vox) respecto a las salidas constitucionales a la crisis catalana.

El PSOE (con el impulso del PSC) acepta la negociación bilateral con el Gobierno independentista de la Generalitat y transige - ide modo táctico?- con ciertos términos simbólicos queridos por el independentismo (vagas referencias al respeto a "la seguridad jurídica" en lugar de a la Constitución, hacer votos por no judicializar la política, así en los dos encuentros "bilaterales" realizados hasta ahora). Ello supone abrirse a reformas políticas graduales que deberían desembocar en una reforma federal del Estado autonómico. Para el PSC, como para Podemos y el nacionalismo vasco, incluso en un federalismo plurinacional, además de asimétrico, que es rechazado por las demás Comunidades Autónomas.

Entre las medidas sugeridas, algunas de ellas ya puestas en marcha por el Gobierno de Sánchez, están las siguientes, ordenadas de menor a mayor alcance político y rango de las normas afectadas: primero, reformas en leyes sectoriales (educación, dando mayor peso a la Generalitat en la determinación de planes de estudio, o leyes penales, con el cambio en la tipificación del delito de secesión), o el impulso de medidas de tipo penitenciario que permitan la vuelta a casa de los condenados, incluso de gracia; segundo, propuesta de reformas legislativas que permitan recuperar opciones del Estatuto de 2006 que fueron declaradas inconstitucionales por razones formales (no ser el Estatuto la norma adecuada para regular materias como la desconcentración territorial del poder judicial o la financiación autonómica); tercero, una reforma del Estatuto de 2006, que debería aprobarse en referéndum y que permitiría volver a las grandes opciones del Estatuto de 2006 (o a las maximalistas de 2005, esto es, las del proyecto de Estatuto aprobado por el Parlamento catalán antes de enviarlo al Congreso). Ahora, con el recurso previo de inconstitucionalidad (reinstaurado en 2015, en reforma de la Ley Orgánica del Tribunal Constitucional), se evitaría el llamado "choque de legitimidades", pues solo se sometería a referéndum el texto aprobado tras la declaración de constitucionalidad, en caso de impugnación ante el Tribunal Constitucional. Esta vía ha sido defendida por 
algunos profesores de Derecho Público, bastantes de ellos cercanos al Partido Socialista. ${ }^{20}$ Por último, se ha formulado la propuesta de reforma constitucional del Estado de corte federal. ${ }^{21}$ La mayoría de las propuestas de reforma constitucional en España inciden en la organización territorial del Estado y no en incorporar a la Constitución una cláusula de secesión..$^{22}$ Esto es, optan por afrontar la crisis secesionista no con una respuesta directa a esta, sino por vía indirecta: una reforma del modelo de Estado.

Los partidos del centro y la derecha han tenido por ahora posiciones más bien reactivas, que han llegado a pedir, en momentos de tensión, una nueva aplicación a Cataluña del artículo 155 de la CE o de la ley de seguridad nacional. Asimismo, se ha rechazado la reforma constitucional (y estatutaria) que pudiera dar cabida a las pretensiones nacionalistas. Por tanto, o bien no se formula una propuesta de organización política del Estado -distinta de la existente (PP)-, o se aboga por la centralización del Estado (Vox) o por un federalismo cooperativo (Ciudadanos). Por el contrario, dichos partidos ponen el énfasis en la debida observancia -por parte de las autoridades catalanas, de la Constitución y del Estado de derecho- y en la existencia de un foro institucional -el Congreso- como sede apropiada para cualquier diálogo político. Este debería centrarse en cuestiones concretas de gestión y competenciales (como sostuvo el Gobierno de Rajoy), no en la discusión sobre la autodeterminación.

En el plano político, parece que, aunque de forma muy frágil, se intenta cada vez más un pacto entre posibilistas de un lado y de otro. Esto, sin embar-

20 Véase el punto 21 del documento Ideas para la Reforma de la Constitución, elaborado por Muñoz Machado et al. (2017, p. 14), en el cual, además, se aboga por abundar en la bilateralidad y por dejar la definición simbólica de Cataluña al Estatuto. Por último, se indica el referéndum a llevar a cabo y el contexto: "Es posible (e incluso constitucionalmente preceptivo) una consulta sobre una ley de Cataluña que adapte el Estatuto, lo reforme y mejore. Incluso consideramos plausible y recomendable que un referéndum de esta clase pueda celebrarse al mismo tiempo que otro de carácter general en el que se pronuncien todos los ciudadanos del Estado sobre la adaptación de la Constitución al nuevo modelo de organización territorial que en este documento propugnamos" (punto 22) (Machado et al., 2017, pp. 14-15). Adviértase que el referéndum previsto en el Estatuto no es, en términos rigurosos, sobre una ley de Cataluña, sino sobre una ley aprobada por el Parlamento catalán y luego negociada y susceptible de ser modificada por las cortes.

21 Así lo formuló el PSOE en la conocida como Declaración de Granada de 2013: Un nuevo pacto territorial: la España de todos (Consejo Territorial, 2013). Se aboga por la reforma constitucional que prevea la constitucionalización del mapa autonómico, la clarificación de la distribución competencial, un Senado territorial, la garantía constitucional de los hechos diferenciales y singularidades, la introducción de un nuevo sistema de financiación autonómica y la creación de mecanismos de cooperación interterritorial. Muñoz Machado et al. (2017) coinciden en lo sustancial y dotan de contenido técnico a estas propuestas del documento citado.

22 Una excepción a ello puede verse en Alaez (2015). 
go, plantea dificultades de distinto tipo: de entrada, de alcanzar los debidos consensos políticos que les den estabilidad y continuidad, al margen de las mayorías del Gobierno de turno. Hoy por hoy, parece muy difícil que el independentismo y los grupos del centro y de la derecha acepten reformas de la Constitución y del Estatuto. Es más que dudoso que ERC se aviniera a una reforma constitucional de corte federal plurinacional o incluso confederal, salvo que incluyera una cláusula de secesión. Y los grupos de centro y de derecha temen que más que una reforma constitucional se abra la puerta a un proceso constituyente, de ruptura con el denominado "régimen de 1978" (como desea Podemos). Esto sucede, además, en un contexto de mayor polarización social respecto a las opciones de reforma del Estado, al aflorar con fuerza posicionamientos políticos ya sea recentralizadores o independentistas, que en 1978 eran muy minoritarios.

Para concluir, la cuestión a dilucidar en los próximos tiempos es el alcance de las propuestas de reforma federal del Estado, bajo las cuales anidan opciones distintas, ya sea de corrección de déficits detectados en la organización y funcionamiento del Estado autonómico en su conjunto en sus cuarenta años de experiencia y de adaptación a las necesidades actuales (exigencias de la Unión Europea, mayor cooperación interterritorial, actuación de la subsidiariedad) o de cambios más profundos. Estos últimos tendrían el propósito de reconducir la actual crisis secesionista y de atender a ciertas demandas de los nacionalismos vasco y catalán, y que suponen establecer una mayor asimetría entre las nacionalidades y las regiones, e incluso el reconocimiento de la plurinacionalidad del Estado (y del bilateralismo consiguiente). En definitiva, si los cambios propuestos son compatibles con la continuidad y la renovación de la nación española como comunidad política o, por el contrario, diluyen los vínculos de unidad, solidaridad y cooperación interterritoriales que se han ido configurando históricamente y que ahora representa el Estado autonómico, sin perjuicio de que puedan lograrse nuevos equilibrios entre pluralidad y unidad, capaces de favorecer a la vez mayor reconocimiento de identidades y mayor integración política.

\section{Bibliografía}

Aguado Renedo, C. (2013). Sobre un eventual referéndum consultivo catalán en el proceso soberanista. Cuadernos de Alzate, (46-47), 27-47. 
Alaez, B. (2015). Constitucionalizar la secesión para armonizar la legalidad constitucional y el principio democrático en Estados territorialmente descentralizados como España. Revista d'Estudis Autonòmics i Federals, (22), 136-183.

Barceló i Serramalera, M., Corretja, M., González Bondia, A., López, J. y Vilajosana, J. M. (2015). El derecho a decidir. Teoría y práctica de un nuevo derecho. Barcelona: Atelier.

Castellà Andreu, J. M. (2014). El problemático encaje constitucional del "derecho a decidir" de Cataluña y sus vías de ejercicio. Percorsi Costituzionali, (3), 807-830.

Castellà Andreu, J. M. (2016). Tribunal Constitucional y proceso secesionista catalán: respuestas jurídico-constitucionales a un conflicto político-constitucional. Teoria y Realidad Constitucional, (37), 561-592.

Consejo Territorial. (6 de julio de 2013). Un nuevo pacto territorial: la España de todos. http:// web.psoe.es/source-media/000000562000/000000562233.pdf.

Consell Assessor per a la Transició Nacional. (2014). Libro Blanco sobre la Transición Nacional de Cataluña. Barcelona: Generalitat de Catalunya.

Closa, C. (2020). A Critique of the theory of democratic secession. En Closa, C., Margiotta, C. y Martinico, G. (Eds.). Between democracy and law. The amorality of secession. Londres: Routledge.

De Miguel Bárcena, J. (2019). Justicia constitucional y secesión. El caso del proceso soberanista catalán. Madrid: Reus.

Ginsburg, T. y Aziz, Z. H. (2018). How to save a constitutional democracy. Chicago: University of Chicago Press.

Jaria i Manzano, J. (2015). La independència com a procés constituent. Consideracions constitucionals sobre la creació d'un Estat català. Revista d'Estudis Autonòmics i Federals, (22), 184-218.

Muñoz Machado, S., Aja Fernández, E., Carmona Contreras, A., De Carreras Serra, F., Fossas Espadaler, E., Ferreres Coma, V., García Roca, J., López Basaguren, A., Montilla Martos, J. A. y Tornos Mas, J. (2017). Ideas para la Reforma de la Constitución. http://idpbarcelona.net/ docs/actual/ideas_reforma_constitucion.pdf.

Pérez Royo, J. (2011). La STC 31/2010 y la contribución de la jurisprudencia constitucional a la configuración de un estado compuesto en España: elementos de continuidad y ruptura, e incidencia en las perspectivas de evolución del estado autonómico. Revista Catalana de Dret Públic, (43), 121-149.

Pons Rafols, X. (2015). Cataluña: Derecho a decidir y derecho internacional. Madrid Reus.

Ridao Martin, J. (2014). La juridificación del derecho a decidir en España. RDP, (91), 91-136.

Rubio Llorente, F. (8 de octubre de 2012). Un referéndum para Cataluña. El Pais.

Rubio Llorente, F. (11 de febrero de 2013). Un referéndum que nadie quiere. El Pais. 
